\title{
An Improved Negative Pressure Wave Method for Natural Gas Pipeline Leak Location Using FBG Based Strain Sensor and Wavelet Transform
}

\author{
Qingmin Hou, ${ }^{1}$ Liang Ren, ${ }^{2}$ Wenling Jiao, ${ }^{1}$ Pinghua Zou, ${ }^{1}$ and Gangbing Song ${ }^{2,3}$ \\ ${ }^{1}$ School of Municipal and Environmental Engineering, Harbin Institute of Technology, Harbin 150090, China \\ ${ }^{2}$ Faculty of Infrastructure Engineering, Dalian University of Technology, Dalian 116024, China \\ ${ }^{3}$ Department of Mechanical Engineering, University of Houston, Houston, TX 77004, USA \\ Correspondence should be addressed to Wenling Jiao; jiaowenling62@163.com
}

Received 24 August 2013; Accepted 27 September 2013

Academic Editor: Ting-Hua Yi

Copyright (C) 2013 Qingmin Hou et al. This is an open access article distributed under the Creative Commons Attribution License, which permits unrestricted use, distribution, and reproduction in any medium, provided the original work is properly cited.

\begin{abstract}
Methods that more quickly locate leakages in natural gas pipelines are urgently required. In this paper, an improved negative pressure wave method based on FBG based strain sensors and wavelet analysis is proposed. This method takes into account the variation in the negative pressure wave propagation velocity and the gas velocity variation, uses the traditional leak location formula, and employs Compound Simpson and Dichotomy Searching for solving this formula. In addition, a FBG based strain sensor instead of a traditional pressure sensor was developed for detecting the negative pressure wave signal produced by leakage. Unlike traditional sensors, FBG sensors can be installed anywhere along the pipeline, thus leading to high positioning accuracy through more frequent installment of the sensors. Finally, a wavelet transform method was employed to locate the pressure drop points within the FBG signals. Experiment results show good positioning accuracy for natural gas pipeline leakage, using this new method.
\end{abstract}

\section{Introduction}

Nowadays, pipelines have become ubiquitous for natural gas transportation. Therefore, natural gas pipelines play a vital role in modern enterprises and economies. However, leakage from natural gas pipelines occurs due to inevitable factors such as pipeline aging, erosion, natural disaster, and third party intrusion. Without proper and immediate fixing, leakage can lead to serious pollution and the danger of explosion due to the poisonous and explosive properties of natural gas. Therefore, effective ways to detect the exact position of leakage accidents are important, so that losses and danger can be greatly reduced.

Over recent years, the number of techniques for leak location of natural gas pipeline has been grown [1]. Presently, methods based on control theory and signal processing are popular, such as the methods based on model detection, pressure gradient, and methods based on negative pressure waves $[2,3]$. With the advantage of quick response speeds, the negative pressure wave method based on pressure sensors is the most widely used leak location technology [4]. However, the installation of pressure sensors required for this method necessitates localized deconstruction of the pipeline. So pressure sensors are usually installed at input and output points of a pipeline and seldom anywhere in between. This leads to large signal attenuation and interference, leading to high rates of false alarms whilst reducing the precision of locating algorithms. In addition, the traditional leak location formula often assumes that the propagation velocity of the negative pressure wave and the velocity of natural gas in the pipeline are constants, even ignoring the velocity of natural gas. This does not match with the actual situation and will inevitably result in a large positioning error. Therefore, research is required into developing an improved method to overcome these shortcomings.

Distributed fiber optical sensors have been widely used for leak detection and location of natural gas pipeline [57]. The simple principle of this method is that a leak in a 
natural gas pipeline will lead to temperature variation or vibration, which can be detected by a distributed fiber optical sensor. The position of leakage point can be obtained by processing the captured signal. Temperature variation or vibration can be caused by other factors such as environment temperature change or random vibration of fiber optical. Thus vulnerable to interference and high false positive rate are main drawbacks of this method.

Fiber Bragg grating (FBG) sensor offers a number of advantages over traditional sensor, including immunity to electromagnetic interference, being light weight and durable, having small size, incorporating multiplexing capabilities, and is easy to install [8-14]. Due to the above attractive application features, FBG sensors have been playing an increasingly important role in the sensing community and have been widely used in structural health monitoring, damage detection, aviation, and other fields [15-21]. However, FBGs have not been extensively adopted in natural gas pipeline leakage location. While a kind of swellable polymer based FBG strain sensor has been proposed for oil pipeline leak detection and location [22]. The main component of natural gas is methane, the chemical properties of methane are very stable, and only a scarce number of polymer types swell when encountering methane. Therefore, this method is not suitable for natural gas pipeline. It is necessary to develop a FBG sensor applicable to natural gas pipelines.

In this paper, firstly, a modified leakage location formula was proposed based on the principle of negative pressure wave location, and Compound Simpson formula and Dichotomy Searching were employed to solve this formula. Secondly, a FBG based strain sensor for collecting the negative pressure wave signals was developed and experimentally tested. Finally, in order to get accurate time difference for that formula, a wavelet transform method was demonstrated to identify the pressure drop point within the FBG signals.

\section{Modified Leak Location Formula}

2.1. Principle of Negative Pressure Wave Leak Location and Traditional Leak Location Formula. When leaks develop in a natural gas pipeline, the gas density near the leaking point will decrease rapidly. This phenomenon results in a negative pressure wave, which propagates through the pipeline from the leak point. Pressure sensors installed upstream and downstream can collect such negative pressure wave signals. According to time difference for detected signals and propagation velocity in the medium, the exact position of the leakage can be calculated [23]. The principle for negative pressure wave propagation is now described, in relation to Figure 1.

In regard to Figure 1, assume that the distance between two sensors is $L$, the propagation velocity of negative pressure wave in the pipeline is $v$, the distance between the leak point and upstream sensor is $x$, the times when the wave is detected by the two sensors are $t_{1}, t_{2}$, and the velocity of natural gas in the pipeline is $u$.

As the pipeline diameter and gas transportation velocity increase, the velocity of natural gas cannot be ignored compared with that of the negative pressure wave. With the velocity of natural gas taken into consideration in our study,

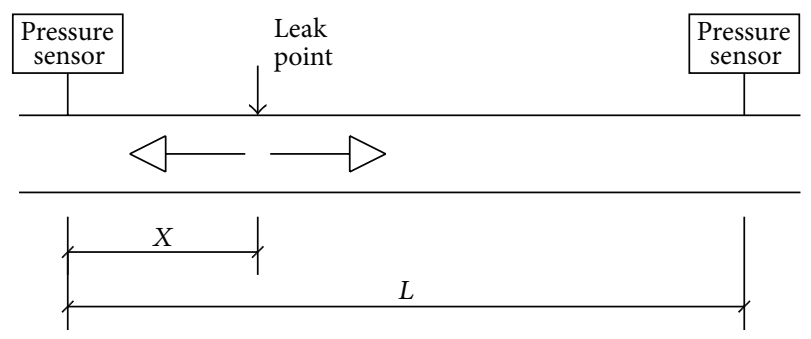

FIGURE 1: Schematic of negative pressure wave propagation.

the relations between the length and time variables can be developed as follows:

$$
\begin{aligned}
& t_{1}=\frac{x}{(v-u)}, \\
& t_{2}=\frac{(L-x)}{(v+u)}, \\
& \Delta t=t_{1}-t_{2} .
\end{aligned}
$$

The distance between the leak point and upstream sensor can be obtained from (1):

$$
x=\frac{1}{2 v}\left[L(v-u)+\Delta t\left(v^{2}-u^{2}\right)\right] .
$$

Equation (2) is the traditional leak location formula.

2.2. Modification of Leak Location Formula. The traditional formula assumes that the propagation velocity of the negative pressure wave, $v$, and the velocity of natural gas in the pipeline, $u$, are constants. In fact, $v$ and $u$ are related with the temperature, pressure, density, and specific heat of the surrounding medium, and the formulas of these two velocities can be obtained by thermal and hydraulics analysis. Treating the propagation velocity of the negative pressure wave and natural gas as variable parameters, the leak location formula can be rewritten as

$$
\begin{gathered}
t_{1}=\int_{0}^{x} \frac{1}{v(x)-u(x)} d x, \\
t_{2}=\int_{x}^{L} \frac{1}{v(x)+u(x)} d x, \\
\Delta t=t_{1}-t_{2} .
\end{gathered}
$$

Since the expressions for $v(x)$ and $u(x)$ are complex, the integral above is not a simple definite integral. Therefore, numerical integral was chosen for determining $t_{1}, t_{2}$. The Compound Simpson formula was employed to calculate the variable integral as given above.

As can be seen from the above equations, if a value for the leak position, $x$, is assumed, the propagation velocity of negative pressure wave $v(x)$ and the velocity of natural gas in the pipeline $u(x)$ can be calculated. Finally, the time difference $\Delta t$ can be determined, from which the leak position $x$ can be improved. This is a recursive formula, as $x$ cannot be solved deterministically. Therefore, a Dichotomy Searching was employed to solve this problem. 


\subsection{The Methods of Solving the Modified Formula}

2.3.1. Compound Simpson Formula. The integral domain $[a, b]$ is divided into $n$ equal parts. The approximate integral value of the function $f(x)$ in this domain can be obtained by

$$
\int_{a}^{b} f(x) d x=\frac{h^{n}}{6} \sum_{i=0}^{-1}\left[f\left(x_{i}\right)+4\left(x_{i+1 / 2}\right)+f\left(x_{i+1}\right)\right],
$$

where $x_{i}=a+i h(i=0,1, \ldots, n)$ and $h=(b-a) / n$, which is the step length.

Equation (6) is the Compound Simpson formula. Using this formula to solve (3) and (4), the time difference $\Delta t$ can be obtained as

$$
\begin{aligned}
\Delta t= & t_{1}-t_{2} \\
= & \int_{0}^{x} \frac{1}{v(x)-u(x)} d x-\int_{x}^{L} \frac{1}{v(x)-u(x)} d x \\
\approx & \frac{h}{6} \sum_{i=x}^{x-1}\left[\frac{1}{v\left(x_{i}\right)-u\left(x_{i}\right)}+4\left(\frac{1}{v\left(x_{i+1 / 2}\right)-u\left(x_{i+1 / 2}\right)}\right)\right. \\
& \left.\quad+\frac{1}{v\left(x_{i+1}\right)-u\left(x_{i+1}\right)}\right] \\
& -\frac{h}{6} \sum_{i=x}^{L-1}\left[\frac{1}{v\left(x_{i}\right)+u\left(x_{i}\right)}+4\left(\frac{1}{v\left(x_{i+1 / 2}\right)+u\left(x_{i+1 / 2}\right)}\right)\right. \\
& \left.+\frac{1}{v\left(x_{i+1}\right)+u\left(x_{i+1}\right)}\right] .
\end{aligned}
$$

2.3.2. Dichotomy Searching. In order to locate the leaking point, Dichotomy Searching was used to determine $x$. Dichotomy Searching is explained below in reference to Figure 2.

As shown in Figure 2, the midpoint $t_{2}$ of domain $\left[t_{0}, t_{4}\right]$ is obtained to calculate $f\left(t_{2}\right)$, then $f\left(t_{0}\right), f\left(t_{2}\right)$, and $f\left(t_{4}\right)$ are compared (in case of $f\left(t_{0}\right)<0, f\left(t_{4}\right)>0$ ).

(1) If $f\left(t_{2}\right)>0$, then $\left[t_{2}, t_{4}\right]$ is rejected, and Dichotomy Searching is going in the domain of $\left[t_{0}, t_{2}\right]$. The next computational point is the midpoint of domain $\left[t_{0}, t_{2}\right]$.

(2) If $f\left(t_{2}\right)<0$, then $\left[t_{0}, t_{2}\right]$ is rejected, and Dichotomy Searching is going in the domain of $\left[t_{2}, t_{4}\right]$. The next computational point is the midpoint of domain $\left[t_{2}, t_{4}\right]$.

In order to use this method to calculate the leak position, a function $f(x)$ is defined as follows:

$$
\begin{gathered}
f(x)=\frac{h}{6} \sum_{i=0}^{x-1}\left[\frac{1}{v\left(x_{i}\right)-u\left(x_{i}\right)}+4\left(\frac{1}{v\left(x_{i+1 / 2}\right)-u\left(x_{i+1 / 2}\right)}\right)\right. \\
\left.+\frac{1}{v\left(x_{i+1}\right)-u\left(x_{i+1}\right)}\right]
\end{gathered}
$$

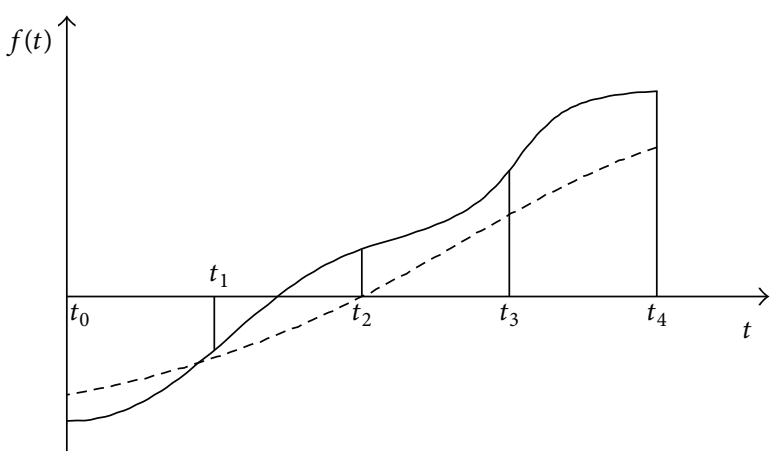

FIGURE 2: Sketch map of the Dichotomy Searching.

$$
\begin{aligned}
-\frac{h}{6} \sum_{i=x}^{L-1}[ & \frac{1}{v\left(x_{i}\right)+u\left(x_{i}\right)} \\
& +4\left(\frac{1}{v\left(x_{i+1 / 2}\right)+u\left(x_{i+1 / 2}\right)}\right) \\
& \left.+\frac{1}{v\left(x_{i+1}\right)+u\left(x_{i+1}\right)}\right]-\Delta t
\end{aligned}
$$

The root of equation $f(x)=0$ is just the leak position, and the flow chart of solving process is shown in Figure 3.

2.4. Critical Factors of Follow-Up Work. From (8), it can be clearly observed that the actual detection time difference plays an important role in leak location. Two critical factors for precise measurements of the time difference have been identified as follows:

(1) sensors that can be easily installed, such that the spacing between sensors can be kept small,

(2) accurate identification of the pressure drop point $\left(t_{1}, t_{2}\right)$ from the sensor pressure trace, as this directly influences the sensitivity and reliability of leakage locating.

The following two sections address these critical factors.

\section{FBG Based Strain Sensor and Experiment}

3.1. Principle of FBG Based Strain Sensor. FBG based strain sensors are wrapped around the wall of a pipeline, as shown in Figure 4. A change in pressure within the pipeline leads to its expansion or contraction with the hoop (circumferential) strain of the pipeline changing accordingly. The FBG strain sensors detect pressure changes within the pipe by sensing the hoop strain. The hoop strain within a pipeline system can be expressed as

$$
\varepsilon_{y}=\frac{\sigma_{y}-v \sigma_{z}}{E}
$$

where $\varepsilon_{y}$ is the pipeline hoop strain, $v$ is the pipeline Poisson ratio, $\sigma_{y}$ is the pipeline hoop stress, $\sigma_{z}$ is the pipeline axial stress, and $E$ is the pipeline elasticity modulus. 


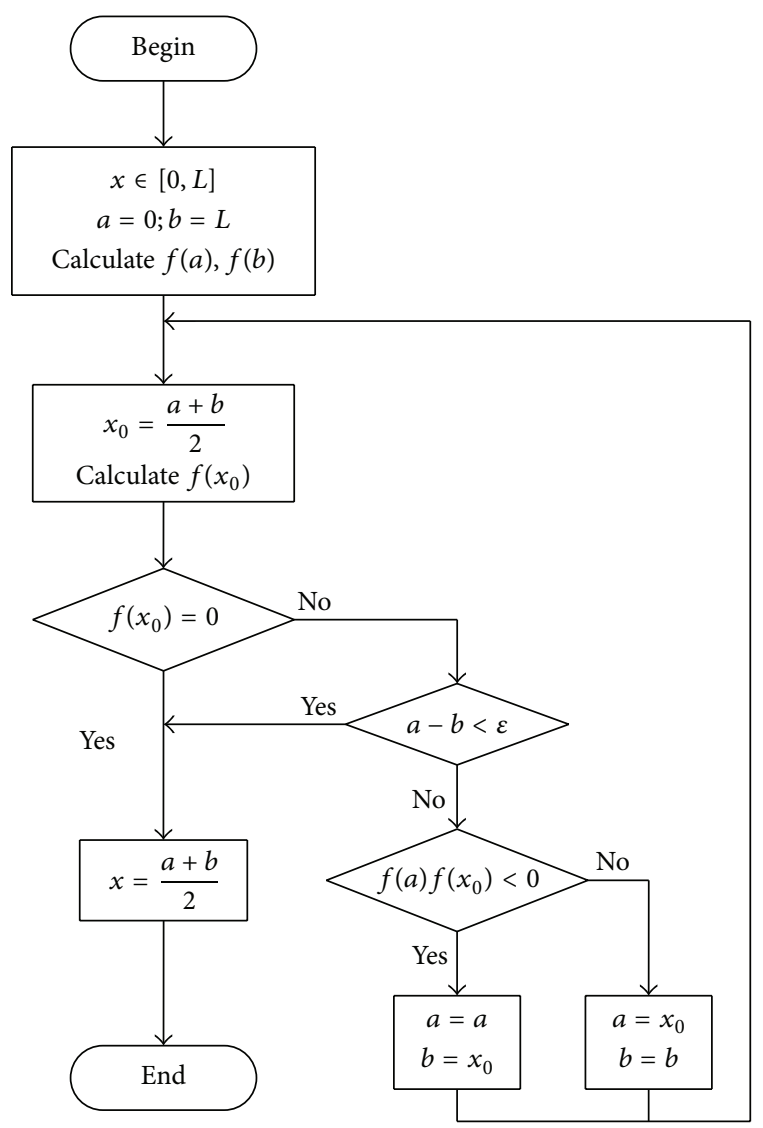

FIGURE 3: Flow chart of solving process.

Using (9), a relationship can be derived to relate the hoop strain with the pipeline pressure and pipe wall thickness. First, it is assumed that the pipeline is infinitely long, so that axial stress can be neglected; that, is $\sigma_{z}=0$. Meanwhile, as $\sigma_{y}=p R / h$, the values for $\sigma_{y}$ and $\sigma_{z}$ can be substituted into (9), which gives

$$
\varepsilon_{y}=\frac{p R}{h E}
$$

where $p$ is the pressure in the pipeline, $R$ is the pipeline internal radius, and $h$ is the pipeline wall thickness. As seen from (10), as the pressure in the pipeline changes, the pipeline hoop strain also changes linearly. Therefore FBG based strain sensors can detect the pressure variation by monitoring the hoop strain of the pipe wall.

3.2. Experimental Setup. The proposed methodology for detecting and locating gas pipeline leakage was tested on an experimental pipeline. The schematic for this gas pipeline is shown in Figure 5(a). Two air tanks and a section of pipeline were used to simulate a realistic gas transfer main. The pipeline in this experiment is made of steel, with a diameter of $273 \mathrm{~mm}$ as frequently used in practice. The pipeline length was $11 \mathrm{~m}$ due to lab space limitations. A leak point was simulated by manually opening valve at locations. As shown in Figure 5(b), a rotameter was located at the leak point for measuring the leak rate, and two FBG strain sensors (L1, L2) were installed.

Briefly, the experimental process followed these steps: First, air is compressed into air tank 1 by air compressor. Air tank 1 plays the role of stabilizing the pressure in the pipeline. Second, when the pressure is stable, a valve is opened to simulate a leak in the pipeline. In the meantime, all the sensors gather data.

3.3. Experimental Results. The signals from FBG based strain sensors were captured to determine their ability to sense negative pressure waves caused by sudden leaks. A leakage was simulated by opening the valve at the leak point as shown in Figure 6(a). Sensors set upstream and downstream collected the hoop (circumferential) strain response, and the pipeline pressure was calculated from this signal. As seen in Figure 6, the pressure was steady before the leak occurred. A sudden pressure drop developed in the waveform that resulted from the leak. Because the duration of the leak was limited, so the pipeline finally returned to a steady but lower pressure due to the loss of gas. Furthermore, the waveforms recorded at L1 and L2 are similar, as shown in Figures 6(a) and 6(b); this was expected due to the propagation of the negative pressure wave on either side of the leak. These results indicate that the FBG based strain sensors can accurately detect the negative pressure wave produced by leakage events.

\section{Identify the Pressure Drop Points Using Wavelet Transform}

4.1. Definition of Wavelet Transform. The wavelet transform method was used to find the sharp transition in the FGB trace, corresponding to the movement of the negative pressure wave passes through the FBG sensor location. This method was used because this method can scale up the signal to find the sharp transition simply.

The definition of wavelet transform for a function $x(t)$ is given in [24-26]

$$
\begin{aligned}
W T_{x}(a, b) & =\int x(t) \psi^{*} \frac{[(t-b) / a]}{\sqrt{a}} d t \\
& =\int x(t) \psi_{a, b}^{*}(t) d t=\left\langle x(t), \psi_{a, b}(t)\right\rangle .
\end{aligned}
$$

In this equation, $a, b$, and $t$ are continuous variables, which is also the reason why (11) is called the continuous wavelet transform. If the wavelet coefficients are computed on all possible scales, the computational burden is large. With the consideration of practical feasibility in numerical computation and simplicity of theoretical analysis, wavelet transforms are normally discretized in practice. The relevant discrete wavelet transform is described by

$$
C_{j, k}=\int_{-\infty}^{+\infty} f(t) \psi_{j, k}^{*}(t) d t=\left\langle f, \psi_{j, k}\right\rangle,
$$




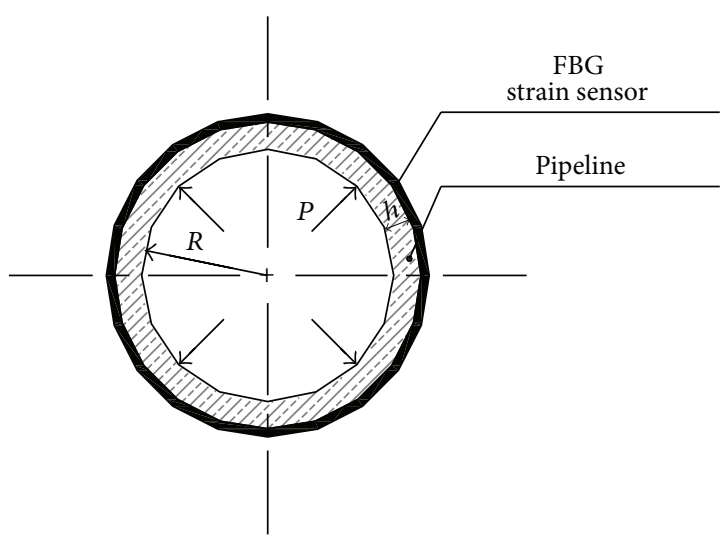

(a)

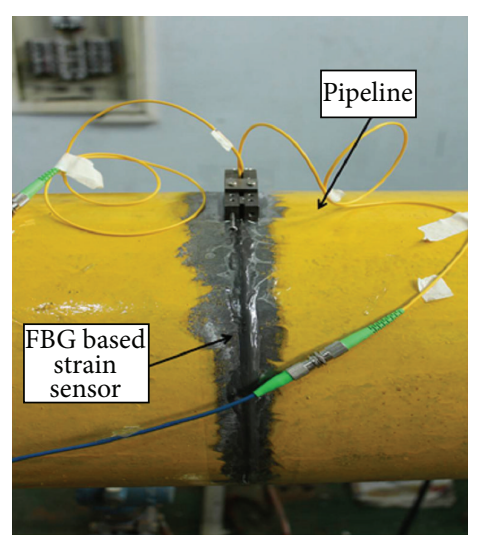

(b)

FIGURE 4: (a) Schematic of a FBG based strain sensor as installed on a pipeline; (b) photo of the sensor installed on an experimental pipeline.

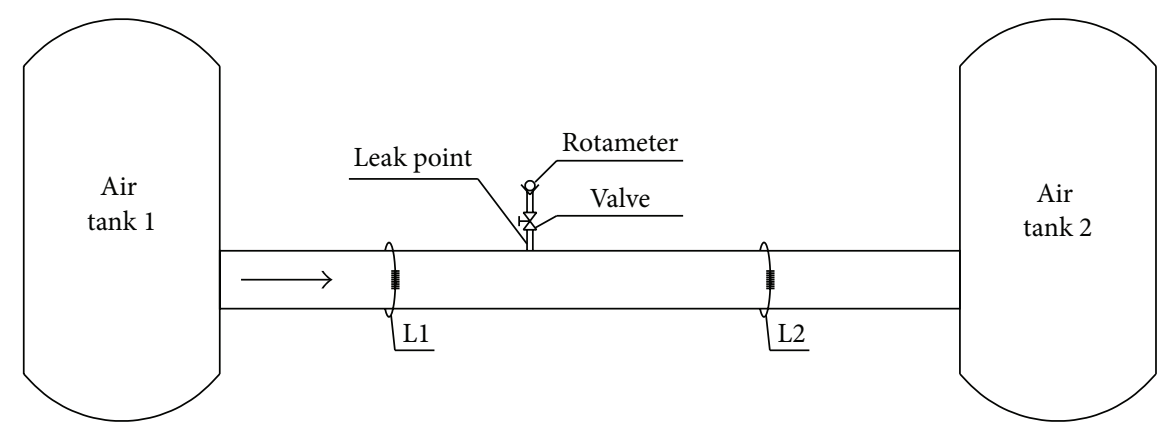

(a)

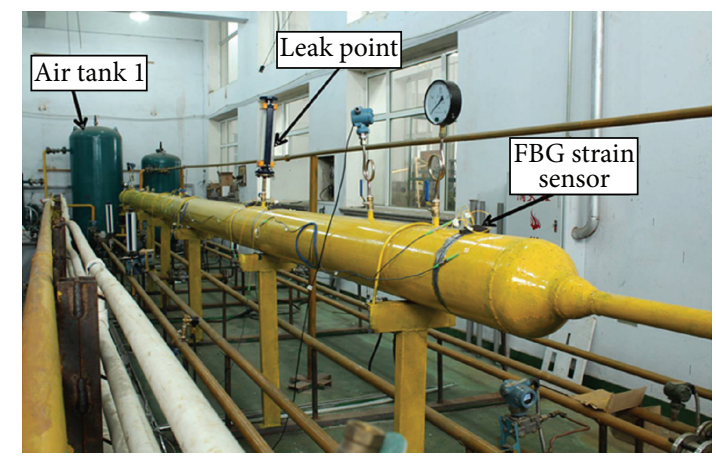

(b)

FIGURE 5: (a) Schematic diagram of experiment setup; (b) photo of experiment setup.

where $\psi_{j, k}(t)$ can be expressed in the following form:

$$
\begin{aligned}
\psi_{j, k}(t) & =\frac{a_{0} \psi\left(t-k a_{0}^{j} b_{0}\right)}{a_{0}^{j}} \\
& =a_{0}^{-1 / 2} \psi\left(a_{0}^{-j} t-k b_{0}\right) .
\end{aligned}
$$
that

In practice, wavelet in (13) is usually dyadic, which means

$$
\psi_{j, k}=2^{-j / 2} \psi\left(2^{-j} t-k\right) j, \quad k \in z
$$

4.2. The Application of Wavelet Transform and Positioning Results. The wavelet transform maxima in modulus on all possible transform scales correspond to the positions where the signals have sharp transition [27]. So the singularity can be obtained by detecting the wavelet transform maxima. Further, the wavelet transform modulus of real singularity is almost fixed value on all scales. In contrast, the modulus of faked singularities is inversely proportional to the scale. Consequently, it is reasonable to determine the real singularity by using this property. However, the detection of singularity is more accurate on a small scale, but this process is likely to be interrupted by noise, As a result, the faked singularity may 


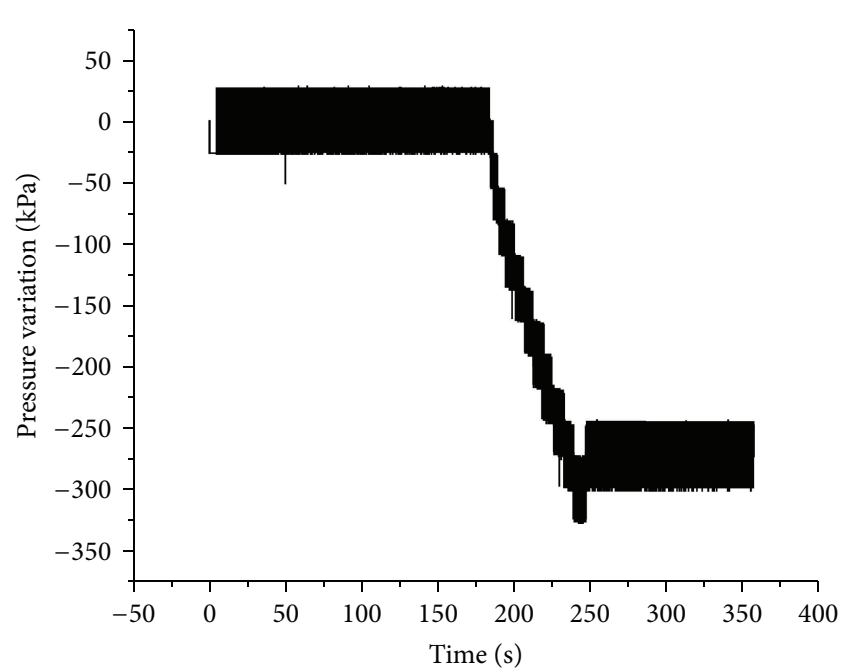

(a)

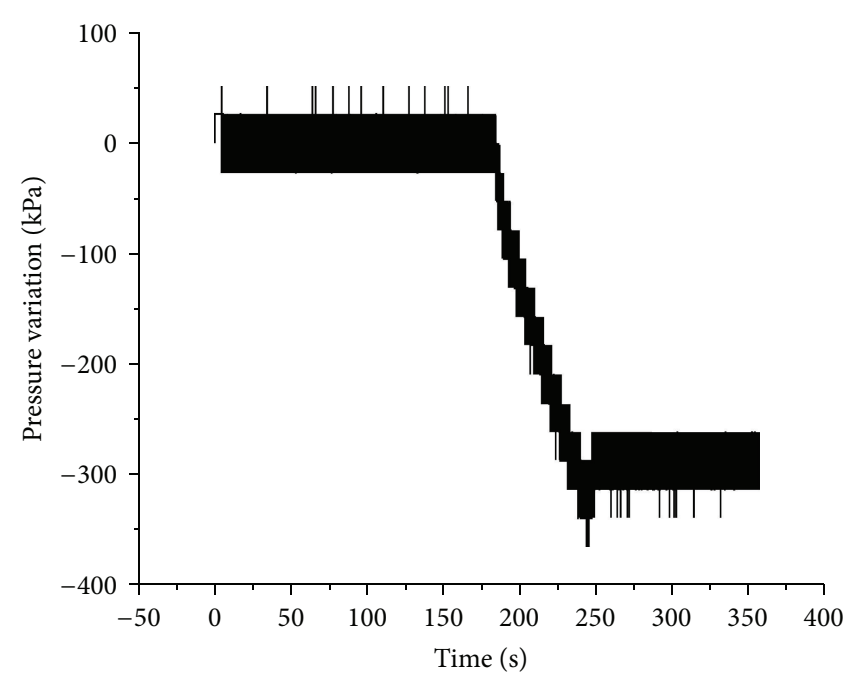

(b)

FIGURE 6: (a) Pressure measured by FBG based strain sensor L1; (b) pressure measured by FBG based strain sensor L2.

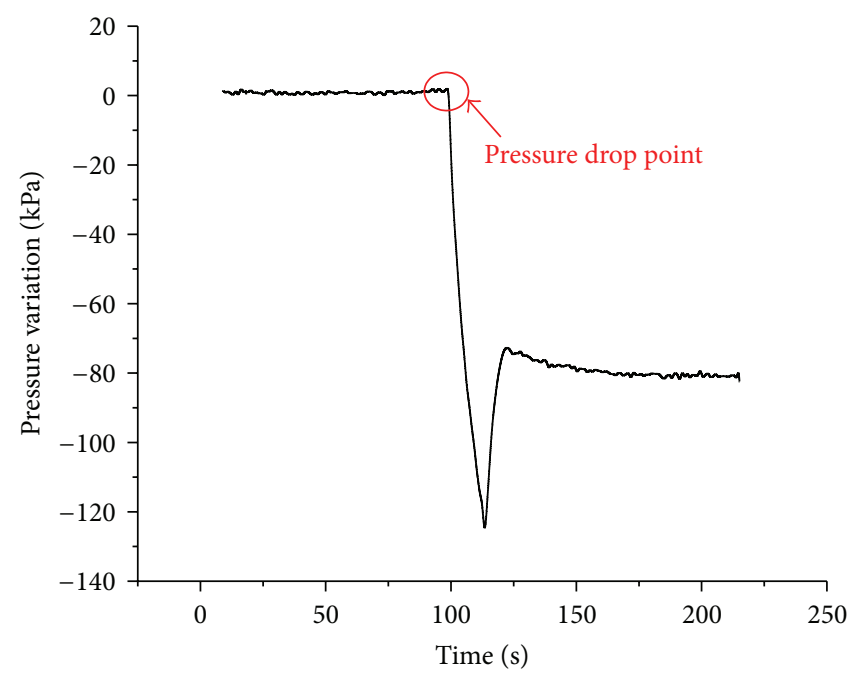

(a)

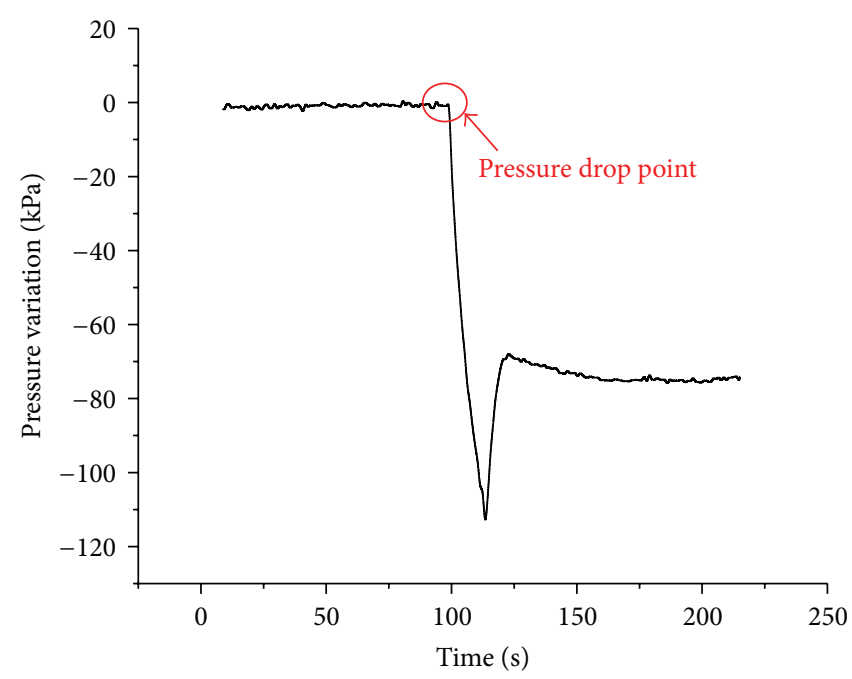

(b)

Figure 7: (a) Denoised pressure signal measured by FBG based strain sensor L1; (b) denoised pressure signal measured by FBG based strain sensor L2.

appear. On the other hand, in the large scale, although noise has little influence on the detection, the deviation between the real singularity and the detective one is large. Since practical pressure signals are mixed with noise even after filtering, it is beneficial to consider multiscale transforms to find the real singularity. Therefore, this study used the following procedure to determine the location of singularities. Firstly, the approximate range wherein the singularity was located was determined by large scales. Secondly, the real singularity was located within this range by taking advantage of the small scales.

In this experiment, the negative pressure wave was produced by opening a valve to simulate a leakage, and the magnitude of the pressure wave was measured by FBG based strain sensors over time. The FBG sensors trace at L1 and L2 were chosen to calculate the leakage position, and the distance between them is $8 \mathrm{~m}$. The denoised signals at $\mathrm{L} 1$ and L2 are shown in Figure 7. The pressure drop points at both $\mathrm{L} 1$ and L2 are marked in Figure 7, and these points were used to determine the time difference using the methodology as describe above.

Figure 8 shows the wavelet analysis of the signal measured at L1 and L2. The pressure drop points in Figure 8 are the singularities. In Figure 8(a) the detail signals at L1 after wavelet transform from scales of 9 to 12 are shown. Similarly, the results from applying wavelet transforms to the pressure signals from L2 are shown in Figure 8(b). When scale $=9$, it can be clearly observed that there are many fake singularities 


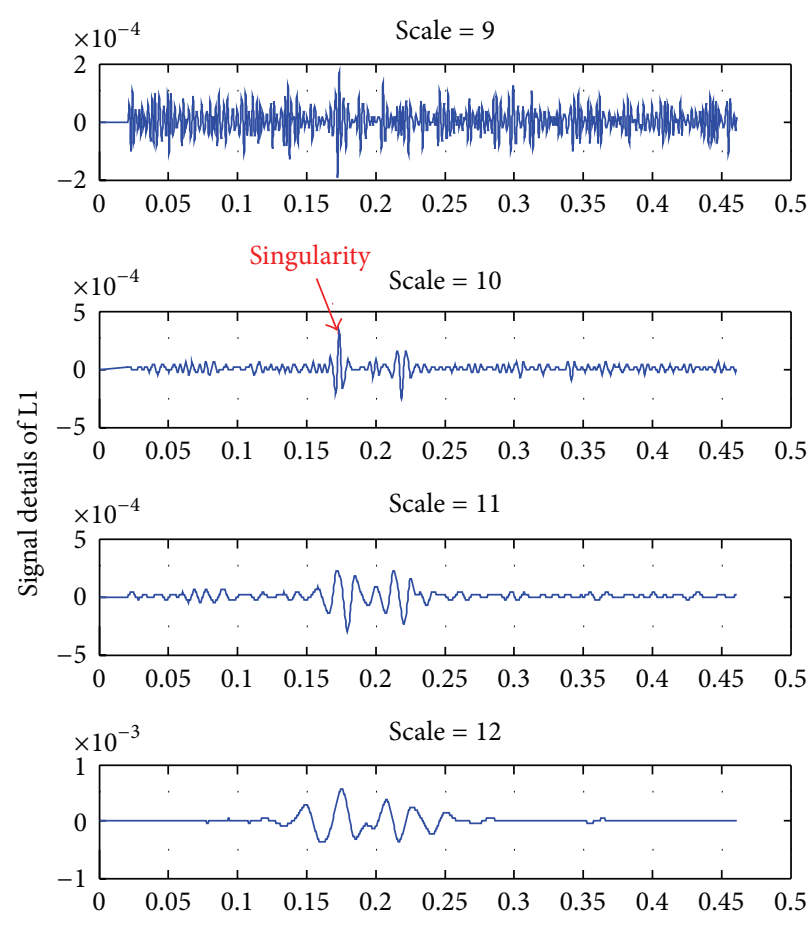

(a)

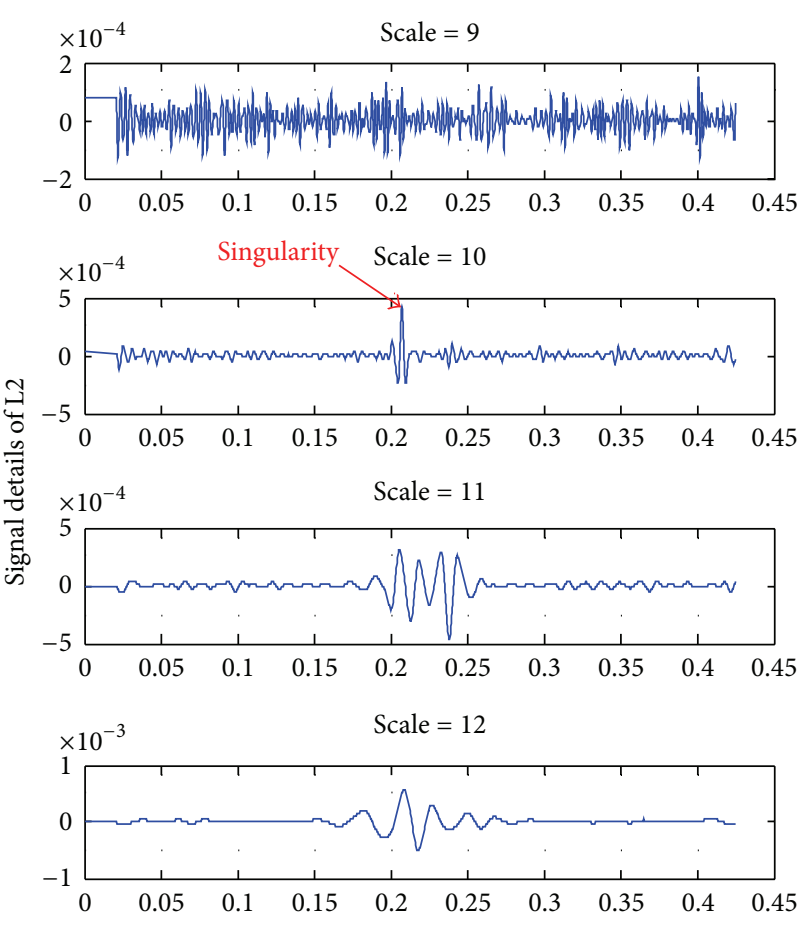

(b)

FIGURE 8: (a) Wavelet analysis of denoised signal measured by L1; (b) wavelet analysis of denoised signal measured by L2.

in the detail signal, and this phenomenon also happens when the scale is less than 8 . On the other hand, as the scale increases, the fake singularities are become less frequent, and the real singularities become more conspicuous, although singularity deviation may happen. Taking advantage of the multiscale transform, the singularities can be identified; therefore, the difference between the arrival time of the negative pressure wave between $\mathrm{L} 1$ and $\mathrm{L} 2$ can be determined. For this case study, this time difference was calculated to be $\Delta t \approx-1.24 \times 10^{-4} \mathrm{~s}$. By substituting this value of $\Delta t$ into (8), the distance between the leak point and L1 was calculated as $x=3.98 \mathrm{~m}$, whilst the actual distance was $3.6 \mathrm{~m}$, yielding an absolute error of $0.38 \mathrm{~m}$ and a relative error of $4.8 \%$. Because the distance between $\mathrm{L} 1$ and L2 in this experiment is very short $(8 \mathrm{~m}), t_{1}$ and $t_{2}$ are very similar, so small deviations in determining $\Delta t$ cause large errors in relative positioning accuracy. However, this limitation would not apply to systems where the distance between L1 and L2 were greater as would be the case in practice. Therefore, the authors have reason to believe that this method can locate the leakage with good accuracy.

\section{Conclusion}

At present, the negative pressure wave method based on pressure sensors is the most widely used leak location technology. In this study, this method is enhanced through incorporating the variation of negative pressure wave and natural gas velocities into the negative pressure wave leak location formula. The Compound Simpson formula and Dichotomy Searching were employed to solve this modified formula. In order to overcome the installation difficulty of traditional pressure sensors, a FBG based strain sensor for collecting the negative pressure wave signals was developed and experimentally tested. Compared to conventional pressure sensors, FBG based strain sensors have favorable properties, such as high sensitivity, cheap cost, and ease of installation. Furthermore, a wavelet transform based method for identifying the pressure drop points within the FBG signals was proposed to calculate the leak position. Utilizing the above methods to calculate the leak position, an absolute error of $0.38 \mathrm{~m}$ was obtained in this experiment. This result demonstrated good positioning accuracy using this improved method.

\section{Acknowledgment}

This work was partially supported by the Science Fund for Creative Research Groups from the National Science Foundation of China under Grant no. 51121005.

\section{References}

[1] R. A. Silva, C. M. Buiatti, S. L. Cruz, and J. A. F. R. Pereira, "Pressure wave behaviour and leak detection in pipelines," Computers and Chemical Engineering, vol. 20, no. 1, pp. S491-S496, 1996.

[2] T.-H. Yi, H.-N. Li, and M. Gu, "Characterization and extraction of global positioning system multipath signals using an improved particle-filtering algorithm," Measurement Science and Technology, vol. 22, no. 7, Article ID 075101, 2011.

[3] T.-H. Yi, H.-N. Li, and X.-Y. Zhao, "Noise smoothing for structural vibration test signals using an improved wavelet 
thresholding technique," Sensors, vol. 12, no. 8, pp. 11205-11220, 2012.

[4] I. R. Ellul, "Advances in pipeline leak detection techniques," Pipes and Pipelines International, vol. 34, no. 3, pp. 7-12, 1989.

[5] B. Vogel, C. Cassens, A. Graupner, and A. Trostel, "Leakage detection systems by using distributed fiber optical temperature measurement," in Smart Structures and Materials 2001: Sensory Phenomena and Measurement Instrumentation for Smart Structures and Materials, Proceedings of SPIE, pp. 23-34, Newport Beach, Calif, USA, March 2001.

[6] E. A. Mendoza, R. A. Lieberman, J. Prohaska, and D. Robinson, "Distributed fiber optic chemical sensors for detection of corrosion in pipelines and structural components," in Nondestructive Evaluation of Utilities and Pipelines II, Proceedings of SPIE, pp. 136-143, San Antonio, Tex, USA, April 1998.

[7] D. S. McKeehan, R. W. Griffiths, and J. E. Halkyard, "Marine applications for a continuous fiber optic strain monitoring system," in Proceedings of the 18th Annual Offshore Technology Conference, pp. 342-345, Houston, Tex, USA, 1986.

[8] K. T. V. Grattan and B. T. Meggitt, Optical Fiber Sensor Technology, vol. 2, Chapman and Hall, London, UK, 1998.

[9] K. T. V. Grattan and B. T. Meggitt, Optical Fiber Sensor Technology, vol. 3-4, Kluwer Academic, Boston, Mass, USA, 1999.

[10] B. Culshaw and J. Dakin, Optical Fiber Sensors, vol. 1-4, Artech House, Boston, Mass, USA, 1988-1997.

[11] A. Dandridge and C. Kirkendall, "Passive fiber optic sensor networks," in Handbook of Optical Fiber Sensing Technology, J. M. Lopez-Higuera, Ed., pp. 433-448, Wiley, New York, NY, USA, 2002.

[12] A. Kersey, "Distributed and multiplexed fiber optic sensors," in Fiber Optic Sensors: An Introduction for Engineers and Scientists, E. Udd, Ed., pp. 325-368, Wiley, New York, NY, USA, 1991.

[13] S. Yin, P. B. Ruffin, and F. T. S. Yu, Fiber Optic Sensors, CRC Press, Boca Raton, Fla, USA, 2nd edition, 2008.

[14] O. S. Wolfbeis, "Fiber-optic chemical sensors and biosensors," Analytical Chemistry, vol. 78, no. 12, pp. 3859-3873, 2006.

[15] S. Yashiro, T. Okabe, and N. Takeda, "Damage identification in a holed CFRP laminate using a chirped fiber Bragg grating sensor," Composites Science and Technology, vol. 67, no. 2, pp. 286$295,2007$.

[16] T.-H. Yi and H.-N. Li, "Methodology developments in sensor placement for health monitoring of civil infrastructures," International Journal of Distributed Sensor Networks, vol. 2012, Article ID 612726, 11 pages, 2012.

[17] H. Tsuda, J.-R. Lee, and Y. Guan, "Fatigue crack propagation monitoring of stainless steel using fiber Bragg grating ultrasound sensors," Smart Materials and Structures, vol. 15, no. 5, article no. 032, pp. 1429-1437, 2006.

[18] D. C. Betz, G. Thursby, B. Culshaw, and W. J. Staszewski, "Advanced layout of a fiber Bragg grating strain gauge rosette," Journal of Lightwave Technology, vol. 24, no. 2, pp. 1019-1026, 2006.

[19] H.-J. Park and M. Song, "Linear FBG temperature sensor interrogation with Fabry-Perot ITU multi-wavelength reference," Sensors, vol. 8, no. 10, pp. 6769-6776, 2008.

[20] N. Takeda, Y. Okabe, J. Kuwahara, S. Kojima, and T. Ogisu, "Development of smart composite structures with small-diameter fiber Bragg grating sensors for damage detection: quantitative evaluation of delamination length in CFRP laminates using Lamb wave sensing," Composites Science and Technology, vol. 65, no. 15-16, pp. 2575-2587, 2005.
[21] T.-H. Yi, H.-N. Li, and H.-M. Sun, "Multi-stage structural damage diagnosis method based on "energy-damage" theory," Smart Structures and System, vol. 12, no. 3-4, pp. 345-361, 2013.

[22] R. M. López, V. V. Spirin, M. G. Shlyagin et al., "Coherent optical frequency domain reflectometry for interrogation of bend-based fiber optic hydrocarbon sensors," Optical Fiber Technology, vol. 10, no. 1, pp. 79-90, 2004.

[23] X. Lu, Y. Sang, J. Zhang, and Y. Fan, "A pipeline leakage detection technology based on wavelet transform theory," in Proceedings of IEEE International Conference on Information Acquisition (ICIA '06), pp. 1432-1437, Shandong, China, August 2006.

[24] I. Daubechies, "The wavelet transform, time-frequency localization and signal analysis," IEEE Transactions on Information Theory, vol. 36, no. 5, pp. 961-1005, 1990.

[25] H. Li, T. Yi, M. Gu, and L. Huo, "Evaluation of earthquakeinduced structural damages by wavelet transform," Progress in Natural Science, vol. 19, no. 4, pp. 461-470, 2009.

[26] T.-H. Yi, H.-N. Li, and M. Gu, "Wavelet based multi-step filtering method for bridge health monitoring using GPS and accelerometer," Smart Structures and Systems, vol. 11, no. 4, pp. 331-348, 2013.

[27] Y. Hao, W. Guizeng, and F. Chongzhi, "Application of wavelet transform to leak detection and location in transport pipelines," Engineering Simulation, vol. 13, no. 6, pp. 1025-1032, 1996. 


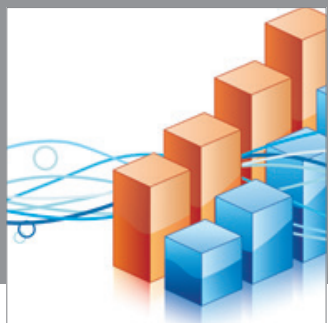

Advances in

Operations Research

mansans

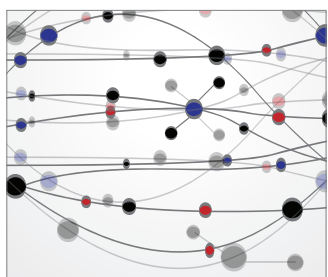

The Scientific World Journal
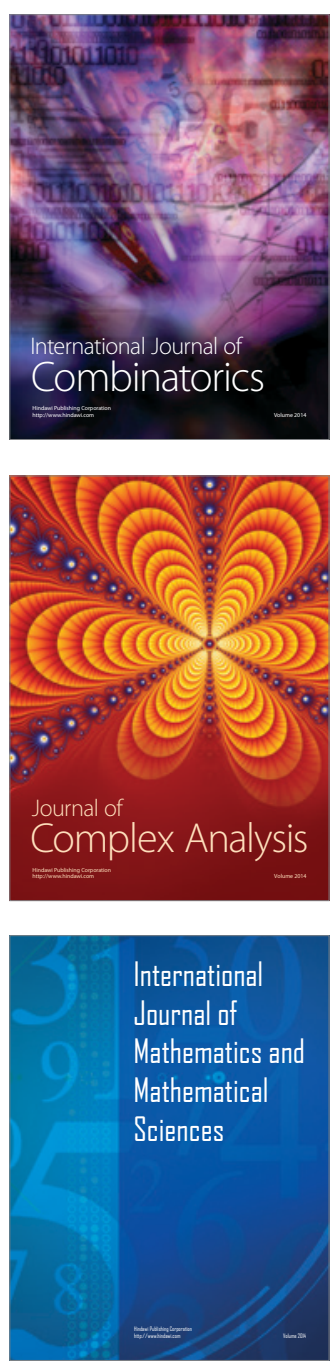
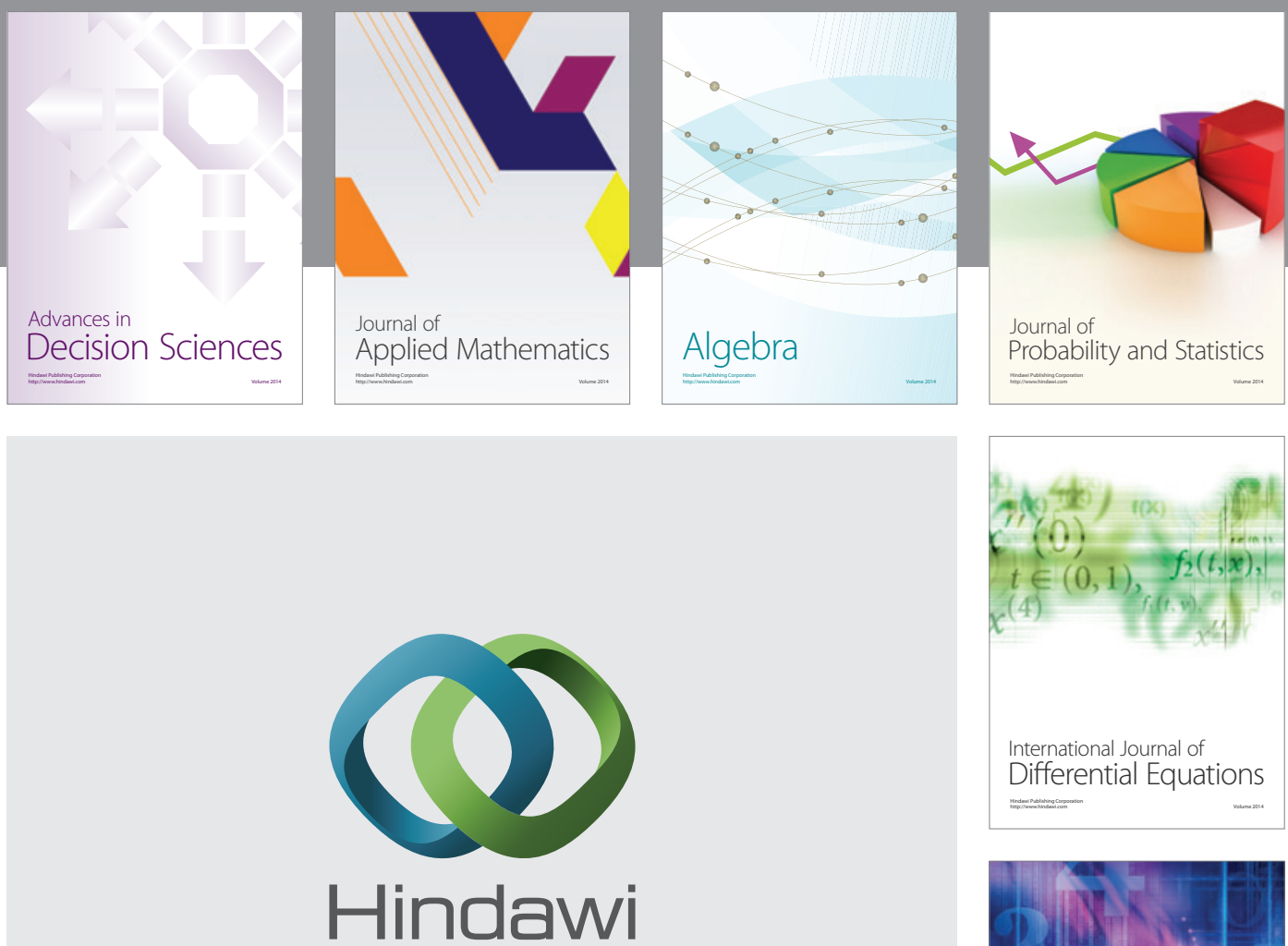

Submit your manuscripts at http://www.hindawi.com
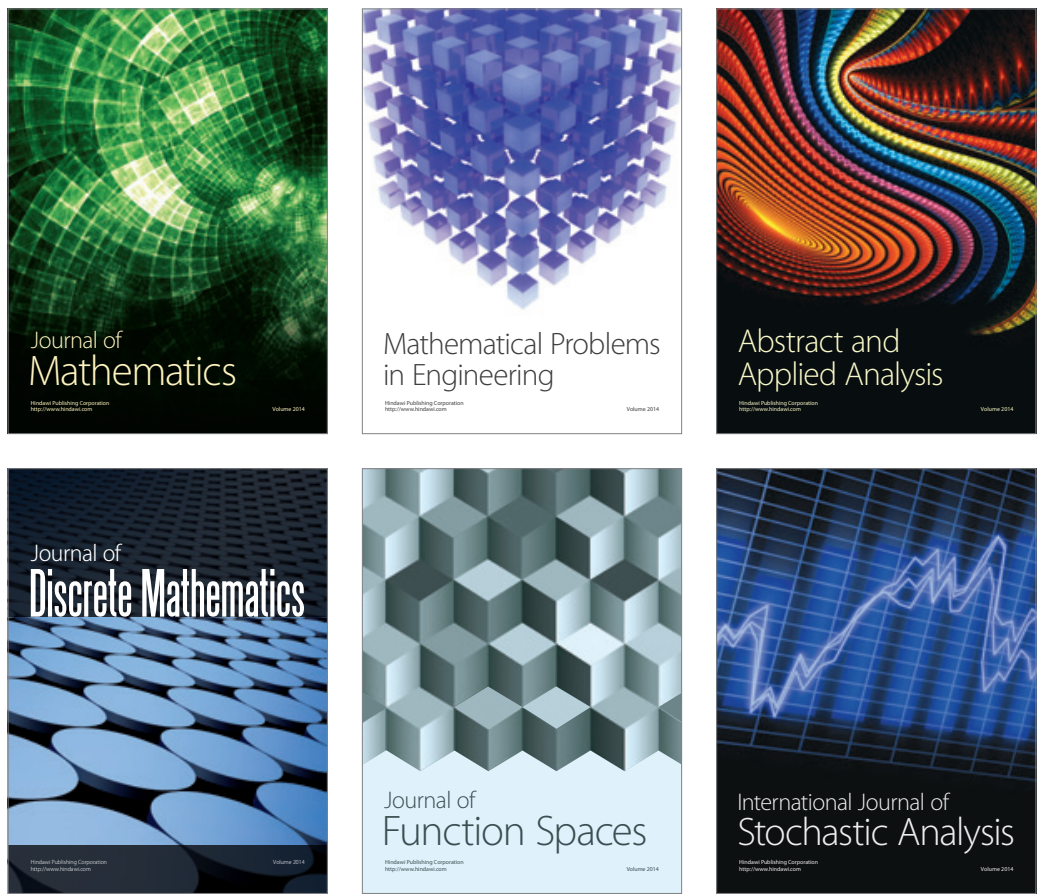

Journal of

Function Spaces

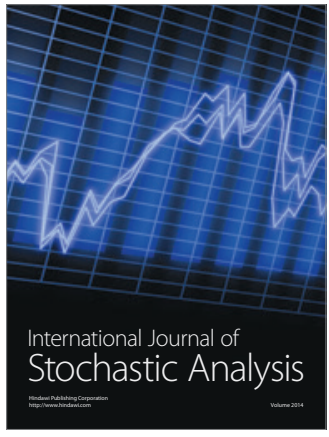

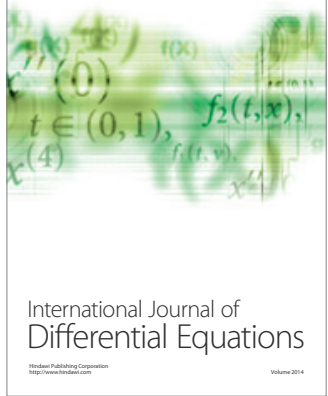
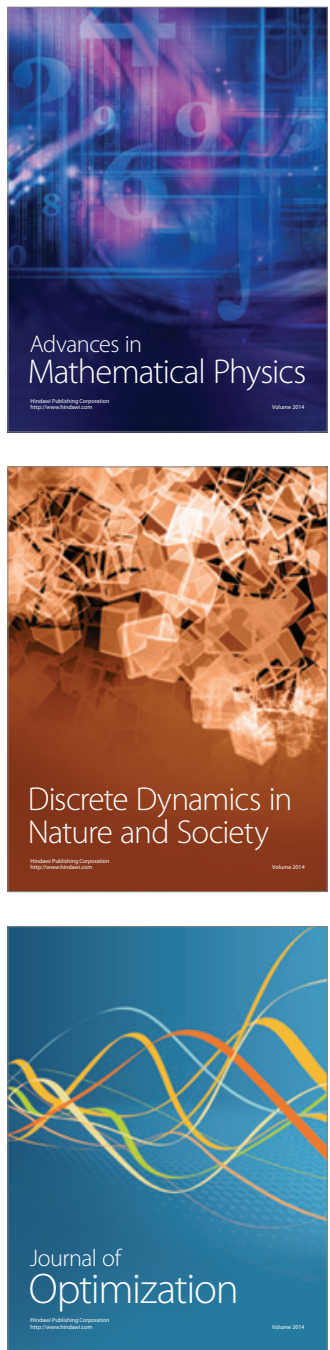